\title{
De los valores a los valores-noticia
}

\author{
From values to the values - news
}

\author{
Amaro La Rosa Pinedo ${ }^{1,}$ \\ 1. Universidad de San Martín de Porres, Lima
}

\section{RESUMEN}

El artículo se inicia con la revisión aleatoria y sintética de algunos criterios teóricos referidos a los valores, pertenecientes a una diversidad de disciplinas y enfoques. Destaca primero las apreciaciones de la Filosofía y continúa con las de la Psicología, la Antropología, la Comunicación y las neurociencias. Nuestra propuesta incide particularmente en el análisis de la relación de los valores con las características de la sociedad. Esto se traduce en rasgos singulares y escalas de valoración propias en cada cultura, así como patrones diversificados de simbolización y evaluación de la realidad. Los valores se vinculan con la interacción de los actores sociales y con el proceso de socialización, en el marco del cual se interiorizan. Se desarrollan después algunos conceptos de valores-noticia, sus enfoques teóricos fundamentales, desde lo planeado por Lippmann. Con las lógicas variantes vinculadas con el paradigma y la experiencia profesional de cada autor, los valores-noticia se entienden en términos generales como líneas de acción para la búsqueda y selección de aquello que por sus cualidades debe considerarse noticia para luego proceder a su procesamiento y difusión. Se comenta su evidente vinculación con la realidad social y con la coyuntura específica que se atraviesa. Finalmente se reseñan los resultados de algunas de las investigaciones realizadas acerca de esta temática en diversas realidades, en la prensa escrita, en las redes sociales y en el discurso sensacionalista.

Palabras clave: Valores, valores-noticia, periodismo

\begin{abstract}
The article begins with the random and synthetic review of some theoretical criteria concerning values, belonging to a variety of disciplines and approaches. First highlights the findings of philosophy and continues with the psychology, anthropology, communication and neurosciences. Our proposal particularly affects the analysis of values relate to the characteristics of the society. This translates into unique traits and scales of assessment in each culture, as well as diversified patterns of symbolization and evaluation of the reality. The values are linked with the interaction of the social actors and the socialization process, in the framework of which are internalized. Some fundamental concepts of values - news, its theoretical approaches, are then developed from planned by Lippmann. With the logic variants linked with the paradigm and the profesional experience of each author, the values - news are understood broadly as lines of action for the search and selection of what for their qualities to be considered news to then proceed with its processing and dissemination. It says its obvious links with the social reality and the specific situation that you go through. Finally the results of some research about this subject several realities are outlined, in the written press, in social networks and sensational speech.
\end{abstract}

Keywords: Values, values - news, journalism.

Historial del artículo:

Recibido, 09 de octubre de 2017; aceptado, 15 de diciembre de 2017; disponible en línea, 05 de enero de 2018

* Licenciado en Psicología, investigador de la Universidad de San Martín de Porres.

Correo: amarolarosa@gmail.com 


\section{INTRODUCCIÓN}

Nos interesamos por la vinculación entre los valores y la construcción de la noticia partiendo de la convicción del papel de los valores como factores fundamentales para la vida del ser humano en sociedad. La evidencia científica ha demostrado fehacientemente que el comportamiento social se ajusta en función de ellos a las expectativas del entorno, de modo tal que configuran un patrón para evaluar la realidad en su conjunto así como para orientar las acciones del individuo en su vida diaria. Los valores se hacen efectivos en todas las culturas y se registran en el lenguaje e inclusive en la comunicación no verbal. Es, por ejemplo, el caso de las tribus no contactadas de la Amazonía. Los especialistas aconsejan no acercárseles para no poner en riesgo la vida; no se conoce su lengua ni sus costumbres, pero sí que valoran negativamente a quienes son extraños a su tribu.

En el marco del proceso de construcción de la noticia (newsmaking) se manejan un conjunto de criterios de valor que involucran filtros para que el medio de comunicación presente su visión de la realidad a partir de lo que identifica como información. No se trata solamente de tecnología, pues al contrario de lo que pudieran pensar los no iniciados, se trata de un complejo proceso que involucra una lectura objetiva de la realidad en la cual los periodistas ejercen un rol activo de toma de decisiones de diverso nivel.

La realidad se presenta de cierta manera, con determinados rasgos que la hacen única y al parecer los medios de comunicación la deberían mostrar tal cual sucede, por cierto en sus términos y códigos específicos. Eso es lo que se enseña habitualmente en las escuelas de periodismo cuando se alude a la denominada objetividad de la información, que supone la presentación a la audiencia de la realidad misma. Brighton y Foy (2007) manifiestan: "En un mundo ideal, las noticias serían verdaderas, válidas, precisas y darían razonada cuenta de lo sucedido. En el mundo real, rara vez es así" (p 163). Agregan que en la práctica del rutinario trabajo periodístico intervienen factores individuales y factores externos de diversa índole, los cuales tienden a modificar la visión de la realidad o inclusive pueden distorsionarla.

\section{Una breve aproximación a los valores}

La importancia de los valores se hace evidente en la medida en que los estudia la Axiología, la rama de la Filosofía que analiza la naturaleza de los valores. Existen en consonancia con ello una serie de conceptos y enfoques que intentan explicar el significado, contenido y alcances de los valores. A continuación algunos criterios de la profusa bibliografía existente.

Mac Leod, Sotirovic y Holbert (1998) definen a los valores de manera sucinta como creencia en que un estado de cosas dado para la sociedad es preferible a su opuesto.

Schwartz (2016), uno de los estudiosos de mayor reputación en esta temática, estima que los valores tienen seis rasgos fundamentales (figura 1).

Estimamos que resulta indispensable contextualizar la problemática de la relación entre valores y sociedad. Sobre el particular, determinados criterios que nos parecen de suma relevancia.

En primer lugar, el punto de vista de Safina (2015) quien señala que los valores se reflejan en el lenguaje de cada cultura. Así por ejemplo, en Rusia la lengua predominante es el ruso, idioma oficial que hablan alrededor de 250 millones de personas. Sin embargo, en ese enorme país que ocupa la novena parte de la tierra firme de nuestro planeta y cruza dos continentes, se hablan unas 250 lenguas, que implican en la

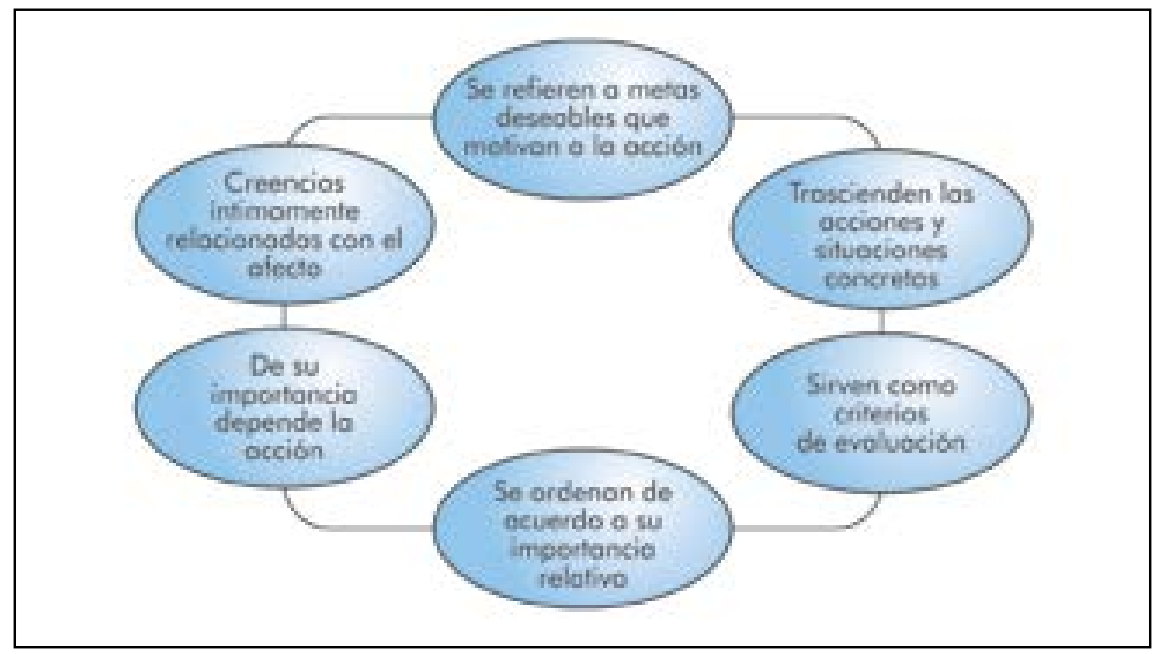

Figura 1. Rasgos fundamentales de los valores. Basado en Schwartz (2016). 
praxis distintas maneras de percibir la realidad y de valorarla. Esta pluriculturalidad hace difícil establecer criterios homogéneos en este particular, tanto más si consideramos como Parihar (2015) que el lenguaje preserva el sistema de valores de una cultura.

Un segundo criterio afirma que los valores permean cada manifestación de la vida social (Rios, 2006), Esto es evidente si consideramos que la propia percepción humana y las modalidades de interacción están condicionadas por la cultura a la cual pertenece el individuo. $Y$ es que los valores están presentes en cada dimensión de lo existente como afirma Cuéllar (2011). Vadi \& Jaakson (2006) sostienen por su parte que los valores tienen como trasfondo a una sociedad concreta, con rasgos predeterminados. Lo ejemplifican a profundidad enfocándose en el valor de la honestidad (figura 2). vista de Hitlin y Piliavin (2004) quienes estiman a los valores como "un ámbito potencialmente propicio en el cual se examinan las influencias recíprocas entre las posiciones socio estructurales y el funcionamiento individual, así como la toma de decisiones" (p. 283), lo cual como veremos, está involucrado necesariamente en los procesos de selección y procesamiento de la información de carácter noticioso.

Acerca de los valores existe abundante información desde la perspectiva filosófica, pero también desde otras vertientes; inclusive desde el punto de vista de la Neurociencia. Así Damasio (2005) afirma que la base de los valores es la homeostasis, en tanto la escala de valores del sujeto funciona como recurso para lograr el equilibrio ante los estímulos positivos o negativos, entre los cuales estarían comprendidos los valores; lo cual por cierto tiene evidentes implicancias con lo psicológico y en lo comunicacional.

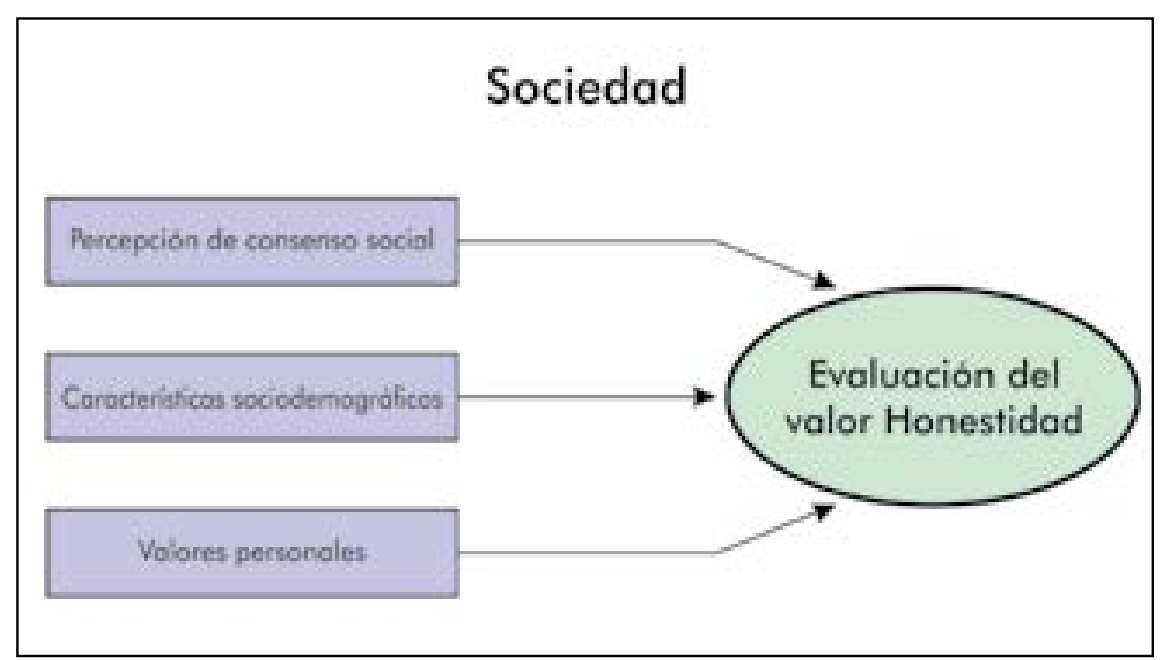

Figura 2. El valor de la honestidad en la sociedad. Basado en Vadi \& Jaakson (2006).

Encontramos aquí coincidencia con el criterio de $\mathrm{Mc}$ Sween (2003), para quien los valores son conjuntos de reglas que prescriben las formas de interacción personal preferidas por una cultura. Pueden aludir tanto a amplias abstracciones como a prácticas específicas e igualmente rutinas perceptuales que involucran formas de "ver el mundo", de percibir lo que nos rodea, las conductas de los demás y los elementos simbólicos. En este sentido se evidencia una concordancia con el enfoque de Prieto (1986).

Lo anteriormente citado no debe suponer, sin embargo, la existencia de una relación causa-efecto mecánica de la sociedad hacia el individuo en el plano valorativo. Sobre el particular, resulta útil entender como Mac Leod et al. (2008), que son los propios individuos quienes buscan por sí mismos sus objetivos personales de comprensión de la sociedad y su autocomprensión. Agregaríamos el punto de
Por su parte, desde un punto de vista etnográfico, Sanmartín (2000) entiende que los valores están íntimamente ligados con la experiencia de la interacción social, que se trasmiten en función de las manifestaciones de conducta de los actores sociales y que se relacionan con asuntos de interés para la sociedad y con la forma apropiada de actuar frente a ellos.

En nuestro contexto tiende a producirse una normalización de la denominada "viveza criolla", que transforma eventualmente en personas socialmente respetables a quienes se han aprovechado de la ingenuidad de otros 0 de los resquicios que dejan la justicia o las leyes y cuya conducta se justifica tácitamente al percibirla como si estuviera dentro de la normalidad, pues "siempre ocurre por razón natural". Algo de esto se expresa cuando en época de elecciones se refieren a cierto candidato, sospechoso 
de conducta dolosa, con la expresión "No importa que robe, lo importante es que haga obras".

En este último caso, cabría cuestionar como Putnam (2004) la dicotomía hecho-valor, entendiendo que uno está imbricado con el otro, dado que al referirnos al sujeto "criollo" estamos aludiendo tanto a la actuación del individuo como refiriéndonos al hecho y al valor que se le adjudica, resultando de facto descalificada dicha aseveración. Esto podemos relacionarlo igualmente con el criterio de Kant cuando afirma que el sujeto produce la ley moral a priori y obra en forma autónoma (Coble, 2012). Así, cuando alguien vive en el interior de un entorno disfuncional, probablemente tienda a percibirlo como normal. Del mismo modo es posible vincularlo con la visión de Alvesson \& Deetz (1996) para quienes los significados que se construyen acerca de la realidad siempre tienen una temporalidad menor que la realidad misma. $Y$ es por ello que, eventualmente, no de percepción de los valores y sus mecanismos reguladores, los cuales a nuestro entender están determinados, al menos en parte, por las condiciones concretas de la vida cotidiana (tabla 1).

A decir de Habermas (1999) "es el entendimiento comunicativo lo que aparece como telos inmanente a la racionalidad" (p. 29). Vale decir, lo que se quiere es lograr la comunicación apropiada trasmitiendo un saber mediante un discurso racional. Esto se produce, desde nuestro criterio mediante un proceso de autorregulación en el sujeto que es factible observar en nosotros mismos cuando hablamos y manejamos conscientemente nuestro discurso para trasmitir lo que deseamos. Habermas asume que si no hay comprensión ello no implica falta de intencionalidad, pues esta es condición sine qua non de la comunicación. No obstante, habría que tomar en cuenta lo que sucede con los sujetos psicópatas y

Tabla 1

Organización psicológica y valores.

\begin{tabular}{lclll}
\hline Nivel & $\begin{array}{c}\text { Valores de } \\
\text { Scheler }\end{array}$ & $\begin{array}{c}\text { Necesidades } \\
\text { de Maslow }\end{array}$ & $\begin{array}{c}\text { Experiencia } \\
\text { psicológica de } \\
\text { los valores }\end{array}$ & $\begin{array}{c}\text { Mecanismo } \\
\text { regulador de } \\
\text { la moralidad }\end{array}$ \\
\hline Biológico & $\begin{array}{c}\text { De los } \\
\text { sentidos } \\
\text { (hedonista) }\end{array}$ & $\begin{array}{l}\text { Biológicas } \\
\text { (fisiológicas, } \\
\text { seguridad) }\end{array}$ & Sentido & Instinto \\
$\begin{array}{l}\text { Simbólico } \\
\text { (Prelógico) }\end{array}$ & De vida & $\begin{array}{l}\text { Psicológicas } \\
\text { (pertenencia, } \\
\text { amor) }\end{array}$ & Experimentado & $\begin{array}{l}\text { Actitudes: } \\
\text { ritual/tabú }\end{array}$ \\
Lógico & Pragmático & $\begin{array}{l}\text { Sociales } \\
\text { (estima) }\end{array}$ & Reconocido & $\begin{array}{l}\text { Pensamiento } \\
\text { lógico: Ley }\end{array}$ \\
Supralógico & $\begin{array}{c}\text { De espíritu, } \\
\text { religioso }\end{array}$ & Autorrealización & "Directamente \\
experimentado" & $\begin{array}{l}\text { Experiencia } \\
\text { directa de } \\
\text { valores }\end{array}$ \\
\hline
\end{tabular}

Fuente: Kokoszka (1991-92). Traducción del autor.

nos percatamos plenamente de lo que supone nuestra realidad, pues la damos como hecho. Encontramos en este particular una marcada coincidencia con Agnes Heller, quien estima que la vida cotidiana está estrechamente unida con el sentido común, en el sentido que representa para cada individuo "la vida misma". Toda persona tiene la convicción que la realidad inmediata en la cual está inmersa es la única posible de aprehender; resultándole compleja la comprensión de lo que está más allá de "su universo", al cual valora de manera positiva y justifica desde su propia óptica que considera como racional (Heller, 1973, 1981).

Kokoszka (1991-92) plantea un modelo evolucionista de niveles de organización psicológica que describe minuciosamente y concibe como referente para la descripción de las maneras concretas de experimentar los valores. Realiza una comparación con las teorías de Scheler y Maslow, así como con la experiencia sociópatas quienes mienten sin ningún reparo; pero allí por cierto también se manifestaría la intención comunicativa del hablante.

\section{Los valores-noticia}

Por lo explicado parecería ser que la problemática de los valores es sobre todo filosófica, con algunos aportes psicológicos, otros sociológicos y antropológicos. No obstante siempre se encuentran nuevos asuntos por investigar en el terreno de la Comunicación, surgen nuevos enfoques teóricos y se detectan nuevas aristas en lo ya estudiado. En nuestro caso cabe anotar que los valores tienen marcada importancia en el procesamiento de la información noticiosa.

Cada día en la redacción de un medio de comunicación se reciben innumerables materiales a través de diversos canales. El proceso de producción de informaciones 
que supone el periodismo debe analizar, valorar, seleccionar y procesar estos datos a fin de lograr el producto final que leeremos, escucharemos o veremos de acuerdo con la naturaleza propia de cada medio o plataforma informativa. Referirnos a cada uno de los aspectos de este procesamiento es una tarea bastante ambiciosa que ya hemos intentado en un trabajo previo (La Rosa, 2013) donde indicamos que uno de los factores que influyen en la construcción mediática de la realidad son precisamente los valores. Nos centraremos en este caso en los valores-noticia.

Para la audiencia, minuto a minuto los medios de comunicación dan a conocer diversas noticias; desde los titulares de primera plana de cualquier diario hasta las noticias publicadas en redes sociales sobre la última pelea prefabricada de dos personajes de la farándula en un reality show. Como dice una antigua frase "de gustos y colores no han escrito los autores"; con cuatro clases de criterios (figura 3).

Bednarek y Caple (2013) estiman que los valoresnoticia pueden enfocarse desde tres perspectivas:

- Material: Potencial de noticiabilidad de un hecho.

- Cognitiva: Creencias de quienes producen las noticias sobre la noticiabilidad de cierto asunto para la audiencia.

- Discursiva: Construcción de la noticiabilidad usando recursos narrativos propios del medio.

Habría que considerar el papel que desempeña el entorno en el reconocimiento y utilización de los valores-noticia. Desde una visión bastante cercana a la que propugnaba en ese entonces el Informe de la Comisión MacBride y refiriéndose a nuestro contexto regional, Reyes (1981) anota: "Las motivaciones para elegir ciertos hechos y darles la categoría de noticia, y

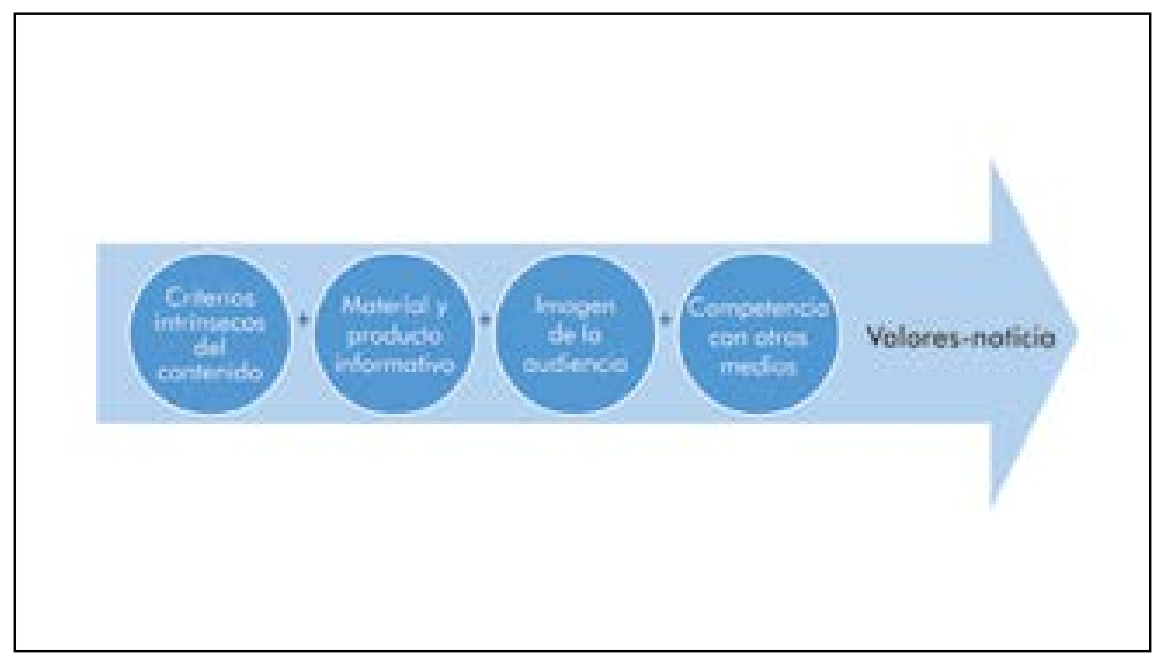

Figura 3. Criterios con los que se relacionan los valores-noticia.

Elaboración del autor. Basada en Wolf (2002)

así pasa cuando las personas tienen la libertad de elegir o descartar noticias, tanto más en esta época de los contenidos hechos a la medida.

A decir de una serie de autores, uno de los primeros aportes teóricos a los valores-noticia fue formulado por Lippmann (1922). Señala que no suele entenderse la diferencia entre la ilimitada complejidad de la sociedad y la limitada naturaleza de las noticias; en consonancia con ello, lo cierto es que la noticia es un aspecto de la realidad que se ha hecho destacar frente a los otros. En este sentido anota Lippmann (1922): "Para que un conjunto de eventos se convierta en noticia, debe contener algo que de modo más o menos evidente sea noticiable" (p. 139).

Para Wolf (2002), los valores-noticia son un componente de la noticiabilidad, vale decir de la posibilidad de que un hecho se transforme en noticia. Estos se relacionan dejando otros de lado, requiere considerar el marco ofrecido por la realidad latinoamericana" (p. 42).

Uno de los criterios sobre valores-noticia que se ha convertido en referente cuando se aborda este tema fue formulado por Galtung y Ruge (1965). Intentamos explicar su criterio de manera sucinta en la tabla 2.

Desde luego habría que ubicar a esta clasificación en el contexto comunicacional de la época en la cual se formuló. A mediados de la década de los sesenta los diarios tenían un rol de primera importancia en la sociedad, constituyendo referentes básicos para la opinión pública. En esa misma época las grandes emisoras internacionales tales como la BBC trasmitían información vía onda corta que solía considerarse confiable y era difundida por los medios más prestigiados que la citaban como su fuente. La televisión por cierto constituía un medio de creciente 
Tabla 2

Valores-noticia según Galtung y Ruge.

\begin{tabular}{ll}
\hline Valor-noticia & \multicolumn{1}{c}{ Descripción } \\
\hline Tiempo & Tiempo que se requiere para que el acontecimiento resulte significativo \\
Amplitud & Tiempo necesitado para que se registre el acontecimiento \\
Claridad & A menor ambigüedad mejor comprensión \\
Significatividad & Representatividad y relevancia cultural \\
Consonancia & Relación con criterios preestablecidos \\
Inesperado & Si sale de lo común merece difundirse \\
Continuidad & Lo que se categoriza como noticia mantiene su vigencia \\
Composición & Los componentes se eligen hasta cierto límite balanceado \\
Referencia & Noticias sobre personas de élite y determinadas naciones \\
Personificación & Las noticias tienden a mostrarse como efectos de acciones individuales \\
Negatividad & Las noticias negativas tienen mayor frecuencia de presentación \\
\hline Fuente: Galtung y Ruge (1965).
\end{tabular}

importancia, que ya mostraba indicios de lo que sería su posterior globalización. Apenas tres años antes había sido lanzado el Telstar, primer satélite de telecomunicaciones que solo permitía un tiempo breve de trasmisión intercontinental dada su órbita elíptica. Un año después sería lanzado el Syncom 3, primer satélite geoestacionario, utilizado para trasmitir las Olimpiadas de Tokio (1964).

Demos un imaginario salto en el tiempo hasta el año 2000. Antes de iniciarse este, la comunidad internacional se preocupaba por el fenómeno del Y2K, mientras que Internet se desarrollaba de modo creciente. Ya se mantenía igualmente activo el periodismo digital, con los recursos que le posibilitaba la tecnología de la época. En este contexto Martini (2000) menciona los siguientes valores-noticia:

1. Novedad.

2. Originalidad, imprevisibilidad e ineditismo.

3. Evolución futura de los acontecimientos.

4. Importancia y gravedad.

5. Proximidad geográfica del hecho a la sociedad.

6. Magnitud por la cantidad de personas o lugares implicados.

7. Jerarquía de los personajes implicados e inclusión de desplazamientos.

La obra de Galtung y Ruge (1965) ha sido analizada críticamente por Harcup y $\mathrm{O}^{\prime} \mathrm{Neill}$ (2001) quienes luego de contrastarla con diversos estudios de especialistas anotan que no se tomaron en consideración las situaciones que más comúnmente se convierten en noticia y en algunos no responden a la propia naturaleza del trabajo periodístico. Proponen una taxonomía de valores-noticia que comprende los siguientes ítems:

1. Elite de poder,

2. Celebridad,

3. Entretenimiento,
4. Sorpresa,

5. Noticias negativas,

6. Noticias positivas,

7. Magnitud,

8. Relevancia,

9. Revisión,

10. Agenda del medio.

A partir de una amplia muestra de titulares extraídos del New York Times y luego de un minucioso estudio, Piotrkowicz, Dimitrova y Markert (2017) reconocen la marcada importancia que tienen en este particular los valores-noticia. Plantean la existencia de los siguientes: prominencia, sentimiento, superlatividad, proximidad, sorpresa y singularidad.

Piotrkowicz, Dimitrova, Otterbacher y Marhert (2017) analizan la popularidad de los valores-noticia en las redes sociales Facebook y Twitter. Para ello analizan y comparan los titulares y metadata de 13806 artículos de The Guardian y 5011 artículos de The New York Times. Definen operacionalmente popularidad a partir del número de likes o de tuits relativos a las noticias. La tabla 3 presenta un resumen de los resultados.

Redondo (2011) realiza una detallada revisión de los valores-noticia en el sensacionalismo. Sostiene que estos comparten una raíz común, se dirigen fundamentalmente a motivar el impacto emocional en la audiencia.

Son para él valores-noticia del sensacionalismo:

1. Malas noticias: violencia, sucesos dramáticos, hechos que creen suspenso y catástrofes.

2. Conflicto y polémica.

3. Erotismo y escándalo sexual.

4. Interés humano transformado en interés morboso.

5. Vida privada. Su impacto sería mayor por cuanto "...por sus cualidades genéricas, recorren transversalmente la información, y no aparecen 
Tabla 3

Popularidad de artículos en Facebook y Twitter

\begin{tabular}{ll}
\hline Valor-noticia & \multicolumn{1}{c}{ Popularidad en Twitter y Facebook } \\
\hline Prominencia & Rasgos más prominentes se correlacionan positivamente con popularidad \\
Sentimiento & Tendencia a favor de titulares negativos y sesgados \\
Superlatividad & Exageración y minimización suelen tener mayor impacto \\
Proximidad & La proximidad cultural suele tener mayor impacto que la geográfica \\
Sorpresa & Impacto de las frases sorpresa \\
Singularidad & Impacto negativo de referencia a titulares recientes \\
\hline Fuente: Piotrkowicz, Dimitrova, Otterbacher y Marhert (2017).
\end{tabular}

circunscritos a secciones concretas" (Redondo, 2011 , p. 208).

Hay que destacar que si bien los valores-noticia configuran una suerte de patrones generales de acción, el conjunto de decisiones que supone la construcción de las noticias no solo depende de aquellos. En este particular coincidimos con Silva (2005) cuando afirma que "la producción de la noticia y de sus características son la resultante de muchos otros factores de noticiabilidad" (p. 99). Tanto más, depende del trabajo del propio periodista, de sus competencias profesionales logradas en el ejercicio de su carrera y de la línea del medio de comunicación.

\section{CONCLUSIONES}

Desde que el periodismo apareció como tal, los valores han sido factores elementales para la construcción de la noticia. No obstante, su naturaleza no siempre ha sido la misma en cada momento histórico ni en cada sociedad. El ideal es por cierto que los valores que se adoptan como estándares para aceptar o rechazar informaciones, sean congruentes con aquellos que conducen al bienestar de la sociedad, en el marco del respeto por la dignidad humana y las normas éticas de un periodismo responsable. Es esta una tarea que está en manos de los periodistas y de las empresas periodísticas pero que también le compete a la academia que debe velar por la formación de profesionales con una sólida formación en valores.

\section{REFERENCIAS BIBLIOGRÁFICAS}

Alvesson, M. \& Deetz, S. (1996). Critical Theory and Postmodernism Approaches to Organizational Studies. En S. Clegg, C. Hardy, \& W. Nord (Eds.), The SAGE Handbook of Organization Studies.Thousand Oaks (pp.191-217). CA: Sage Publications.

Bednarek, M. y Caple, H. (2013). Delving into the Discourse: Approaches to News Values in
Journalism Studies and Beyond. Working Paper. Oxford: Reuters Institute for the Study of Journalism. Brighton, P. y Foy, D. (2007). News values. London: SAGE Publications.

Coble, D. (2012). Ley moral, moralidad, y bien en sí en Kant (un estudio lógico-conceptual de la filosofía moral de Kant). El Búho (revista electrónica de la Asociación Andaluza de Filosofía). Recuperado de http://elbuho.aafi.es/buho10/coble2.pdf

Cuéllar, H. (2011). El ser y la esencia de los valores: Una axiología para el siglo XXI. México D.F.: Trillas.

Damasio, A. (2005).The neurobiogical grounding of human values. En J. Changeux, A. Damasio, W. Singer, y W. Christen (Eds), Neurobiology of human values (pp. 47-56). Heidelberg, Berlin: Springer Verlag.

Habermas. J. (1999). Teoría de la acción comunicativa, I. Santafé de Bogotá: Ediciones Santillana.

Galtung, J. y Ruge, M. (1965). The structure of foreign news. Journal of Peace Research, 2(1), 64-91.

Harcup. T. y O'Neill, D. (2001). What is news? Galtung and Ruge revisited, Journalism Studies, 2(2), 261 280.

Heller, A. (1973). Hipótesis para una teoría marxista de los valores. Barcelona: Ediciones Grijalbo.

Heller, A. (1981). Sociología de la vida cotidiana. Barcelona: Península.

Hitlin, S. y Piliavin, J. (2004) Values: Reviving a dormant concept, Annual Review of Sociology, 30, 359-93. doi: 10.1 146/annurev.soc.30.012703.110640

Kokoszka, A. (1991-92). On the psychology of experiencing values: A supplement to the evolutionary level model of the nain states of conciousness Imagination, cognition and personality, 11(1), 75-84.

La Rosa, A. (2013). Construcción de la agenda mediática: Una mirada al interior de la comunicación. Lima: Universidad de San Martín de Porres.

Lippmann, W. (1922). Public opinion. New York: Harcourt, Brace and Company, Inc.

Mac Leod, J., Sotirovic, M., y Holbert, R. (1998). Values as sociotropic judgements influencing communication patterns. Communication Research, 25(5), 453-485.

Mc Sween, T. (2003). Value-based safety process: Improving your safety culture with behavior-based 
safety. Hoboken, New Jersey: John Wiley \& Sons, Inc.

Martini, S. (2000) Periodismo, noticia y noticiabilidad. Buenos Aires: Norma.

Parihar, U. (2015). The world beyond the words: An axiological comparative study of zoonyms of Russian and Hindi, Russian Journal of Linguistics Viestnik Rudn, 3, 54-71

Piotrkowicz, A., Dimitrova, V., y Markert, K. (2017) Automatic extraction of news values from headline text. In Proceedings of the Student Research Workshop at the 15th Conference of the European Chapter of the Association for Computational Linguistics (pp. 64-74). Valencia, Spain: Association for Computational Linguistics. Recuperado de http://eprints.whiterose.ac.uk/1 10978/7/EACLautomatic-extraction news.pdf

Piotrkowicz, A., Dimitrova, V., Otterbacher, J., y Marhert, K. (2017). The Impact of news values and linguistic style on popularity of headlines on Twitter and Facebook. Proceedings of the Second International Workshop on News and Public Opinion (767774). Montreal, Canada: Association for the Advancement of Artificial Intelligence. Recuperado de https://www.aaai.org/ocs/index.php/ICWSM/ ICWSM17/paper/download/15775/14900

Prieto, D. (1986). Voluntad de verdad y voluntad de espectáculo. Quito: Ciespal.

Putnam, H. (2004). El desplome de la dicotomía hecho-valor y otros ensayos. Barcelona: Ediciones Paidós Ibérica.

Redondo, M. (2011). El sensacionalismo y su penetración en el sistema mediático español (tesis doctoral). Facultad de Filosofía y Letras, Universidad de Valladolid, España.
Reyes, F. (1981). El concepto de noticia en América Latina: valores dominantes y perspectivas de cambio. En A. Da Costa, P. Chopra, y F. Reyes, Valor de las noticias y principios de Comunicación Intercultural. París: UNESCO.

Rios, L. (2006). El fundamento axiológico de las relaciones internacionales y de las Constituciones modernas. En Anuario de Derecho Constitucional Latinoamericano, tomo 2 (pp. 1371-1403). Montevideo: Fundación Konrad Adenauer. Recuperado de http://www.kas.de/wf/doc/55881442-4-30.pdf

Safina, D. (2015). Prospects for steady comparison research in the axiological aspect (Using the example or Russian, English and Tatar languages). The Social Sciences, 10(7), 1844-1847.

Sanmartín, R. (2000). Etnografía de los valores. Teoría de la Educación, 12, 129-141.

Schwartz, S. (2016). Basic individual values: sources and consequences. In T. Brosch, y D. Sander (Eds.), Handbook of value perspectives from Economics, Neuroscience, Philosophy, Psychology, and Sociology (63-84). New York: Oxford University Press.

Silva, G. (2005). Para pensar criterios de noticiabilidade. Estudos em Jornalismo e Mídia, 2(1), 95107.

Vadi, M. y Jaakson, K. (2006). The importance of value honest: Determining factors and some hints to ethics. University of Tartu Working Paper, 43. Recuperado de https://dx.doi.org/10.2139/ ssrn. 888808

Wolf, M. (2002). Teorias da comunicação. Lisboa: Presença. 